\title{
The application of "Omics" to accelerate precision medicine in Supportive Care in Cancer
}

\author{
Stephen Sonis ${ }^{1,2} \cdot$ Jai Patel $^{3} \cdot$ Fredrick D. Ashbury $^{4,5,6}$
}

Received: 16 August 2021 / Accepted: 24 August 2021 / Published online: 21 September 2021

(c) The Author(s), under exclusive licence to Springer-Verlag GmbH Germany, part of Springer Nature 2021

The desirability of applying precision medicine to the treatment of cancer has been of ongoing and expanding importance. Advances in molecular biology and genomics have enabled the discovery of specific somatic mutations, which are often strongly associated with targeted treatment response and have been pivotal in catalyzing a departure away from the "one size fits all" approach that has dominated cancer therapy [1]. It became apparent that histologically similar cancers could vastly differ in their responses to a particular regimen. While oncologic precision initiatives focused on tumor control have led to improvements in cancer survival, the myriad of toxicities associated with anti-cancer drugs and radiation has also provided an opportunity for further individualizing care [2]. Just as individual's tumor responses varied widely, so have patient's toxicity profiles. Moreover, while many of these toxicities bear the same name as those associated with standard chemotherapy drugs, the behaviors are proving to be different and therefore require alternative mitigation strategies.

It has been a consistent observation that phenotypically indistinguishable patients may be vastly different in their susceptibility to a drug's or radiation's effects. A critical toxicity presenting in one patient may be completely absent in another person [3]. Historically, we have largely approached

Stephen Sonis

ssonis@pesclinical.com

1 Division of Oral Medicine, Brigham and Women's Hospital and the Dana-Farber Cancer Institute, Boston, MA, USA

2 Primary Endpoint Solutions, 360 Second Avenue, Waltham, MA 02451, USA

3 Department of Cancer Pharmacology \& Pharmacogenomics, Levine Cancer Institute, Atrium Health, Charlotte, NC, USA

4 VieCure, Denver, CO, USA

5 Department of Oncology, University of Calgary, Calgary, Alberta, Canada

6 Dalla Lana School of Public Health, University of Toronto, Toronto, Ontario, Canada this observation in a reactive way: administer treatment, watch and wait, and then react to mitigate symptoms. Or conversely, play the odds. Rather than try to discern differential risk, treat every patient with an intervention under the assumption that the likelihood of a toxicity justifies the physiologic and economic cost of treating a low-risk cohort. And finally, in treated patients, physicians have largely assumed that all patients respond to a given therapy in a similar way. Unfortunately, these approaches have resulted in high rates of dose-limiting toxicities, which in the curative setting can be profoundly important.

Alternatively, there has been increasing enthusiasm to develop methods which can accurately predict both an individual's toxicity risk profile and the likelihood that a specific intervention might be effective in mitigating a specific toxicity [2]. Risk prediction has often been associated with treatment intensity — more radiation, more toxicity; higher doses of a cytotoxic agent, more toxicity; concomitant chemoradiation and you hit the toxicity jackpot. But these associations are not, in fact, the same for everyone. It has become clear that factors associated with patient-related variables that impact biological response pathways, drug metabolism, and the microbiome in a confluent and interactive way are critical to determining toxicity risk and patients' responses to therapy.

The recognition that patients' genomes can markedly affect toxicity risk was noted years ago with the findings that DPD [4] and TPMT [5] deficiency were associated with high levels of fluoropyrimidine- and thiopurine-induced toxicity, respectively. Since then, multiple examples have been noted including UGT1A1 and irinotecan toxicity [6], NUDT15 and thiopurine toxicity [7], MTHFR [8] and SLCO1B1 [9] and methotrexate toxicity, CEP72 and vincristine toxicity [10], and many more. These discoveries paved the way for the application of pharmacogenomics as a conduit for toxicity risk prediction. But the number of patients affected by mutations impacting cancer drug metabolism is small in comparison to the numbers of individuals who have markedly 
symptomatic or cancer therapy-limiting toxicities. Nonetheless, variations in pharmacogenes can also impact response to several non-oncologic treatments, dozens of which are used as supportive therapies in cancer patients (e.g., antidepressants, antiemetics, antifungals, and opioids) [11]. Novel, albeit expensive, immunotherapies and targeted treatments, coupled with supportive care, are potentially financially toxic and deleterious to successful outcomes, especially if done in the absence of a proper assessment of patient risk.

In its broadest sense, "multi-omics" influences most patients' systemic responses to both drugs and radiation. While one patient might have a robust biologic response to a drug or radiation challenge, the response to the same challenge might be tempered by genomics in someone else. And while genomics has largely been the headliner, it would be naïve to believe that individual genes act independently or that cells and their controlling mechanisms are not impacted by extrinsic elements like the microbiome and epigenome. The bottom line is that there are many moving parts that determine risk and response (the interactome). Clinical factors alone are important predictors of toxicity risk too, especially in vulnerable populations like the elderly (e.g., Cancer and Aging Research Group score) [12], or manifestations of cancer-related disease states like thrombosis (e.g., Khorana Risk Score) [13]. Notably, risk is dynamic and creates fluidity over time. How does a patient's toxicity risk change over time? What biological, clinical, and behavioral factors influence risk? Is a patient's response to a drug or radiation fixed or variable? Many questions, limited answers.

But we have come a long way in better understanding the potential role of omics in supportive cancer care. To date, much of the research (both pre-clinical and clinical) in this area has been dispersed across many journals. With the initiation of a new dedicated section, it is our goal to make Supportive Care in Cancer the preferred platform for manuscripts which address questions associated with precision medicine applicable to cancer regimen-related toxicities and improve clinical practice by applying personalized strategies to mitigate adverse outcomes.

\section{References}

1. Hyman DM, Taylor BS, Baselga J (2017) Implementing genomedriven oncology. Cell 168:584-599

2. Patel JN, Papachristos A (2015) Personalizing chemotherapy dosing using pharmacological methods. Cancer Chemother Pharmacol 76:879-896

3. Patel JN (2015) Cancer pharmacogenomics: implications on ethnic diversity and drug response. Pharmacogenet Genomics 25:223-230

4. Diasio RB, Johnson MR (1999) Dihydropyrimidine dehydrogenase: its role in 5-fluorouracil clinical toxicity and tumor resistance. Clin Cancer Res 5:2672-2673

5. McLeod HL, Krynetski EY, Relling MV, Evans WE (2000) Genetic polymorphism of thiopurine methyltransferase and its clinical relevance for childhood acute lymphoblastic leukemia. Leukemia 14:567-572

6. Innocenti F, Undevia SD, Iyer L et al (2004) Genetic variants in the UDP-glucuronosyltransferase $1 \mathrm{~A} 1$ gene predict the risk of severe neutropenia of irinotecan. J Clin Oncol 22:1382-1388

7. Relling MV, Schwab M, Whirl-Carrillo M et al (2019) Clinical Pharmacogenetics implementation consortium guideline for thiopurine dosing based on TPMT and NUDT15 genotypes: 2018 update. Clin Pharmacol Ther 105:1095-1105

8. Zhao M, Liang L, Ji L et al (2016) MTHFR gene polymorphisms and methotrexate toxicity in adult patients with hematological malignancies: a meta-analysis. Pharmacogenomics 17:1005-1017

9. Lopez-Lopez E, Martin-Guerrero I, Ballesteros J et al (2011) Polymorphisms of the SLCO1B1 gene predict methotrexate-related toxicity in childhood acute lymphoblastic leukemia. Pediatr Blood Cancer 57:612-619

10. Stock W, Diouf B, Crews KR et al (2017) An Inherited genetic variant in CEP72 promoter predisposes to vincristine-induced peripheral neuropathy in adults with acute lymphoblastic leukemia. Clin Pharmacol Ther 101:391-395

11. Patel JN, Olver I, Ashbury F (2021) Pharmacogenomics in cancer supportive care: key issues and future directions. Support Care Cancer. https://doi.org/10.1007/s00520-021-06451-y

12. Magnuson A, Sedrak MS, Gross CP et al (2021) Development and validation of a risk tool for predicting severe toxicity in older adults receiving chemotherapy for early-stage breast cancer. J Clin Oncol 39:608-618

13. Khorana AA, Kuderer NM, Culakova E, Lyman GH, Francis CW (2008) Development and validation of a predictive model for chemotherapy-associated thrombosis. Blood 111:4902-4907

Publisher's note Springer Nature remains neutral with regard to jurisdictional claims in published maps and institutional affiliations. 